\title{
Influence of Surfactant Concentration in the Emulsions on the Process of Oleophilic/Hydrophobic Porous Medium Imbibition
}

\author{
Olga S. Shtyka and Jerzy P. Sęk
}

\begin{abstract}
The spontaneous imbibition has been a subject of the scientific interest being a background process for numerous industrial technologies and occurring in the natural environment. In literature the experimental and theoretical results regarding this phenomenon describe the media imbibition with single-phase liquids and the relation between the process rate and media characteristics. The imbibition of oleophilic/hydrophobic porous structures with two-phase liquids, only one phase of which was wetting, is an objective of the current publication. The main purpose is to estimate the influence of both the dispersed phase concentration and surfactant fraction on the discussed process. The imbibition rate was investigated during model experiments with stabilized oil-in-water emulsions, which had dispersed phase concentrations of $10 \mathrm{vol} \%, 30 \mathrm{vol} \%$, and $50 \mathrm{vol} \%$. The prepared emulsions differed by the fraction of the added surfactant, i.e. $1 \mathrm{vol} \%, 2 \mathrm{vol} \%$, and $5 \mathrm{vol} \%$. The obtained results allowed to conclude that at the $h_{i m} \geq 0.02 \mathrm{~m}$, the dispersed phase concentration and viscosity decreased versus the height. However, the raise of the surfactant fraction caused the increase of mass and height of the imbibed emulsions in porous medium. Moreover, it provided the increase of viscosity and the change of emulsions behaviour as a liquid.
\end{abstract}

Index Terms-Emulsion, imbibition, porous medium, surfactant.

\section{INTRODUCTION}

The spontaneous imbibition is referred as a liquid transport in porous structures, which is driven by the capillary suction-pressure. It appears on pores walls during their wetting by a penetrating liquid, if an adhesion force of liquid molecules exceeds the force of their mutual attraction [1]-[4]. The imbibition is known as a phenomenon occurring in the natural environment, i.e. hydrological regime in a soil matrix, migration processes in geology; plants seeds imbibition with water, etc. Nowadays, it is applied in numerous industrial spheres: in environmental protection (viz. oil and its derivatives recovery, absorption technology); in chemical and material engineering (viz. percolation of the antifungal

Manuscript received March 12, 2016; revised September 29, 2016. The study was funded in framework of the Foundation Program for Young Scientists in the Lodz University of Technology, Grant No. 501/10-34-2-1015.

O. S. Shtyka was with the National Aviation University, Kyiv 03058, Ukraine. She is now with the Lodz University of Technology, Lodz 90-924, (e-mail: olga.shtyka@edu.p.lodz.pl).

J. P. Sęk is with the Lodz University of Technology, Lodz 90-924. He was with the Delft University of Technology, 2628 CD Delft, Netherlands and the University of Groningen, $9700 \mathrm{AB}$ Groningen, Netherlands (e-mail: jerzy.sek@p.lodz.pl). emulsions into concrete constructions), as well as in pharmacology and medicine.

The experimental data on the spontaneous imbibition presented in literature can be divided into the following groups: study of the relation between the process rate (i.e. mass/height change) and time [1], [5]-[10]; study of the dependence between the characteristics of porous structure and the imbibition rate [1], [8]-[10]; and study of the effect of penetrating liquid characteristics on this process [5]-[7]. Majority of the presented results has been related to the imbibition with single-phase liquids [1]-[3], [5]-[11], while the issues concerning multiphase liquids, viz. emulsions, still have not been fully clarified and described. It connects with the complexity of the mentioned process.

Emulsions as thermodynamically metastable systems, in practice can be stabilized by emulsifiers or solid particles [12]-[16], or due to reducing of the droplets size of the inner phase [17]-[19]. However, the adding of emulsifying agents is currently the most common method used for emulsions stabilization. This explains a great deal of the results regarding the influence of different emulsifiers on emulsions stability, and their mechanical and morphological properties, e.g. size distribution of droplets, their shape, dispersity, and others [12]-[15], [20]-[22].

The sphere of our scientific interest is the processes of multiphase liquids transport in porous media. Concretely, this publication provides a discussion and an analysis of the experimental results on the influence of the stabilization agent fractions and the dispersed phase concentrations on a process of porous structure imbibition. The estimated values were the changes of mass and height of the imbibed emulsions over time; the rate of the imbibed mass increase with a rise of the height of an emulsion penetration as well as the variation of the dispersed phase concentration during the imbibition process. In addition to the above, the correlation between a surfactant fraction and a viscosity of the prepared emulsions was investigated.

\section{EXPERIMENTAL PART}

\section{A. Materials}

In these experiments, stabilized oil-in-water emulsions were used as wicking liquids, which dispersed phase behaved as a wetting one, while absolutely non-wetting liquid represented its continuous phase. The emulsions were obtained by means of mechanical stirring during $600 \mathrm{~s}$ at $23 \pm 1^{\circ} \mathrm{C}$. They were prepared ranging from 10 to $50 \mathrm{vol} \%$ of 
the dispersed phase concentration. Moreover, the investigated emulsions differed by fractions of the added emulsifying agent $\left(\varphi_{s}\right)$, which were equal to 1 vol\%, 2 vol\% and 5 vol\%.

The oily component was represented by a refined vegetable oil produced by EOL Polska Sp.z.o.o., Poland. The commercial surfactant Rokacet $\mathrm{O} 7$ was used as a non-ionic emulsifier to stabilize the prepared emulsions. Its hydrophilic-lipophilic balance value equated 10.6. The surfactant was produced and obtained from PCC Exol SA, Poland. The physicochemical properties of the emulsions components are represented in Table I.

TABLE I: PHYSICOCHEMICAL PROPERTIES OF BASIC COMPONENTS

\begin{tabular}{ccccc}
\hline \hline & & \multicolumn{3}{c}{ Physicochemical properties } \\
\cline { 3 - 5 } Type of & Component & Density, & Viscosity, & Surface \\
basic & composition & $\rho$ & $\eta$ & tension, $\gamma$ \\
component & & $\mathrm{kg} / \mathrm{m}^{3}$ & $\mathrm{mPa} \cdot \mathrm{s}$ & $\mathrm{mN} / \mathrm{m}$ \\
\hline Dispersed & Vegetable & 954.0 & 56.3 & 32.2 \\
phase & oil & \pm 1.7 & \pm 0.4 & \pm 1.3 \\
Surfactant & Ethoxylated & 908.0 & 50.2 & 36.0 \\
& oleic acid & \pm 2.7 & \pm 0.6 & \pm 1.8 \\
\hline \hline
\end{tabular}

The porous medium was represented in a form of stripes, which were cut from a non-woven polypropylene sheet obtained from Sintac ${ }^{\circledR}-$ Polska Sp.z o.o.j.v., Poland. Each sample had the same size as a height $h$ of $0.16 \mathrm{~m}$, a width $a$ of $0.035 \mathrm{~m}$, and the mean thickness of $1.9 \times 10^{-3} \mathrm{~m}$.

The used polypropylene sheets belonged to the type of oleophilic/hydrophobic sorbents. According to the experimental data, the sorption capacity of vegetable oil with viscosity of $56.3 \mathrm{mPa} \cdot \mathrm{s}$ was $24.3 \mathrm{~g} / \mathrm{g}$.

The average porosity of the investigated medium was equal to 0.93 .

\section{B. Procedure of the Experiment}

The dependence between the concentration of the added emulsifier and the rate of the imbibition process, the changes of the dispersed phase concentration and the viscosity of an imbibed emulsion versus the height of its penetration in a sorbent were investigated experimentally. The used installation is represented schematically in Fig. 1.

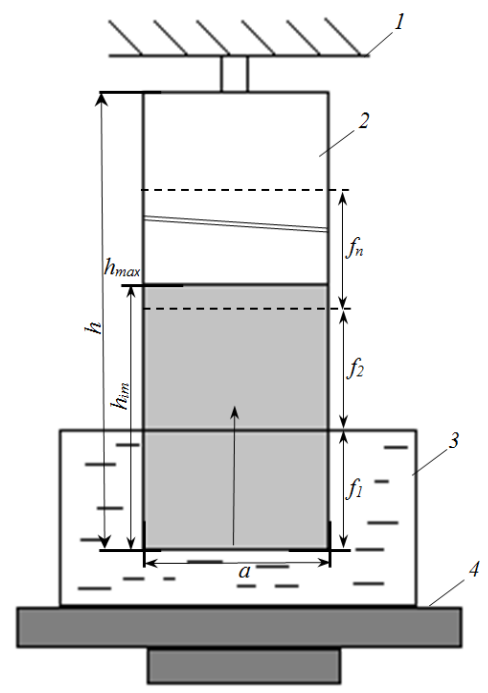

Fig. 1. Scheme of the used equipment:

1 - fixator of a porous material; 2 - stripe of a porous material; 3 - reservoir with the investigated liquid; 4 - balance.
Each of the prepared polypropylene stripes (2) was dipped into a beaker (3) with $200 \mathrm{ml}$ of an emulsion. The contact square of material surface was $\sim 1.3 \times 10^{-4} \mathrm{~m}^{2}$. This method has been described in detail elsewhere [23].

The changes of the height of an imbibed liquid front, i.e. $h_{i m}$, and mass $m_{i m}$ versus time $t_{i m}$ were followed till the time when the process achieved a state of equilibrium. The moment when the investigated parameters became unalterable, was denoted as maximal time of the imbibition, $t_{\max }$. After that, a sorbent stripe was removed from the beaker and its soaked part was cut into fragments $f_{n}$, as shown in Fig. 1. The size of fragments was of a height of $0.02 \mathrm{~m}$ and a width of $0.035 \mathrm{~m}$. Each sample was put into a ceramic dish and the imbibed emulsion was washed out with $10 \mathrm{ml}$ of distilled water.

In the obtained mixture, the dispersed phase concentration was measured by means of the nephelometrical method using the optical analyzer TurbiscanTM LAB (Formulaction, France). The application of this method has been reported precisely elsewhere [24].

\section{Analytical Methods}

The density of the investigated liquids was measured using the picnometric method. The viscosity changes related to increasing of the surfactant and inner phase concentrations were determined by means of a rotational viscometer Rheometer RC 20 (Rheotec, Germany). The shear rate values varied and were in a range of $80-1401 / \mathrm{s}$. The surface tension was measured with a tensiometer KRÜSS K100 (Krüss GmbH, Germany). All experiments were conducted at a temperature of $23 \pm 1^{\circ} \mathrm{C}$ and atmospheric pressure.

\section{RESULTS AND DISCUSSION}

\section{A. Viscosity Changes with Increase of Surfactant Concentration}

According to the obtained data, the increase of a surfactant concentration influenced on viscosity of the investigated emulsions and their behavior as a liquid. The results of the viscosity tests are represented in Fig. 2.

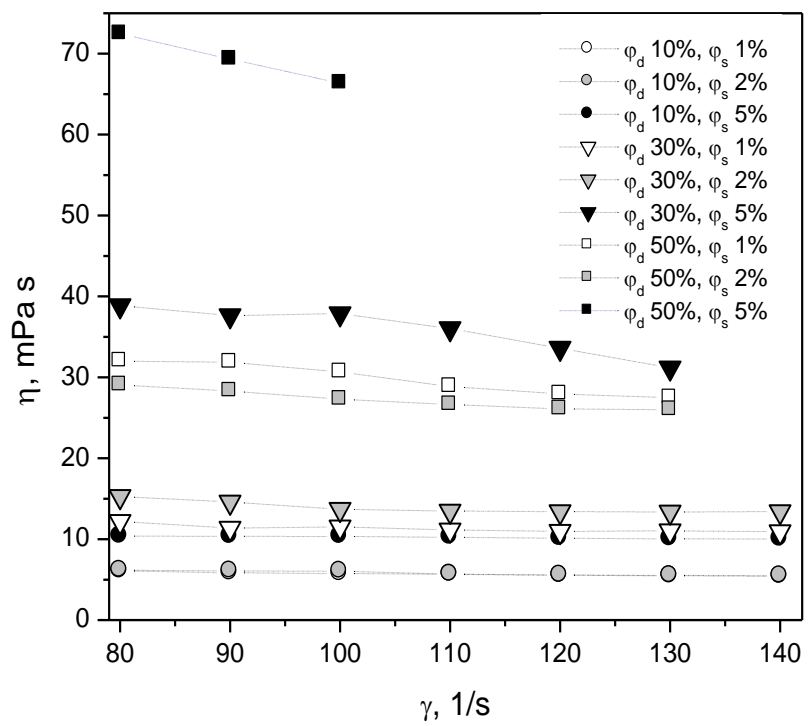

Fig. 2. Changes of viscosity versus increasing of the surfactant concentration at different shear rates. 
The viscosity values were constant at different shear rates for all $10 \%$ emulsions, and $30 \%$ emulsions, but only with $\varphi_{s}$ of $1 \mathrm{vol} \%$ and 2 vol\%. As illustrated in Fig. 2, the obtained data were approximated by straight lines in this case and it means that the investigated dispersions represented Newtonian liquids. However, others behaved as non-Newtonian shear thinning liquids, and consequently, the increase of the shear rate caused the decreasing of viscosity value. For example, in case of $30 \%$ emulsion with $\varphi_{s}=5 \mathrm{vol} \%$, it reduced from $38.9 \mathrm{mPa} \cdot \mathrm{s}$ for $\gamma=801 / \mathrm{s}$ till $31.2 \mathrm{mPa} \cdot \mathrm{s}$ for $\gamma=130 \mathrm{1} / \mathrm{s}$.

The phenomenon of viscosity change as a result of the shear rate increase was observed for all 50\% emulsions. The higher was a surfactant concentration in an emulsion, the more indicative was the observed decrease (Fig. 2).

To conclude, an emulsifier concentration influenced significantly on viscosity of the investigated two-phase liquids. Moreover, the emulsions with higher concentrations of the inner phase and stabilization agent, exhibited properties of non-Newtonian liquids.

\section{B. Changes of Imbibition Rate with Increase of Surfactant Concentration}

The experimental results showed that the process of porous media imbibition with emulsions depended on a fraction of the added surfactant and this revealed to be a variation of the height and mass of imbibed liquids.

The data concerning the maximal height of dispersions penetration in sorbent samples are shown in Table II.

TABLE II: VALUES OF MAXIMAL HEIGHT OF EMULSIONS PENETRATION

\begin{tabular}{cccc}
\hline \multirow{2}{*}{$\begin{array}{c}\text { Dispersed phase } \\
\text { fraction, } \varphi_{d}\end{array}$} & \multicolumn{3}{c}{ The value of height $h_{\max }, \mathrm{m}$} \\
\cline { 2 - 4 } & $\varphi_{s}=1 \mathrm{vol} \%$ & $\varphi_{s}=2 \mathrm{vol} \%$ & $\varphi_{s}=5 \mathrm{vol} \%$ \\
\hline $10 \mathrm{vol} \%$ & 0.081 & 0.091 & 0.101 \\
$30 \mathrm{vol} \%$ & 0.065 & 0.080 & 0.083 \\
$50 \mathrm{vol} \%$ & 0.040 & 0.063 & 0.070 \\
\hline \hline
\end{tabular}

The maximal obtained mass of the emulsions imbibed in the non-submerged part of sorptive material, are presented in Table III.

TABLE III: VALUES OF MAXIMAL IMBIBED MASS OF EMULSIONS

\begin{tabular}{cccc}
\hline \hline \multirow{2}{*}{$\begin{array}{c}\text { Dispersed phase } \\
\text { fraction, } \varphi_{d}\end{array}$} & \multicolumn{3}{c}{ The value of mass $m_{\max }, \mathrm{kg}$} \\
\cline { 2 - 4 } & $\varphi_{s}=1 \mathrm{vol} \%$ & $\varphi_{s}=2 \mathrm{vol} \%$ & $\varphi_{s}=5 \mathrm{vol} \%$ \\
\hline $10 \mathrm{vol} \%$ & 0.0015 & 0.0016 & 0.0024 \\
$30 \mathrm{vol} \%$ & 0.0009 & 0.0012 & 0.0022 \\
$50 \mathrm{vol} \%$ & 0.0004 & 0.0011 & 0.0012 \\
\hline \hline
\end{tabular}

The increase of an emulsifier fraction caused a raise of the imbibed emulsion mass in all investigated cases. For 50\% emulsions, the value of imbibed mass was 3 times higher at $\varphi_{s}=5$ vol\% comparing to the emulsions with the lowest surfactant fraction, i.e. 1 vol\%. Such ratio was equaled to 2.5 for $30 \%$ emulsions and 1.6 for $10 \%$, respectively (Table III). The same tendency was observed for the height of emulsions fronts (Table II). As an example, the results concerning the height variation versus time for $10 \%$ and $50 \%$ emulsions are plotted in Fig. 3-Fig. 4.

In case of $10 \%$ emulsions, it increased from $0.081 \mathrm{~m}$ $\left(\varphi_{s}=1 \mathrm{vol} \%\right)$ to $0.101 \mathrm{~m}\left(\varphi_{s}=5 \mathrm{vol} \%\right)$, i.e. by $25 \%$. For $30 \%$ emulsions, the difference of the fronts height was equal to $\sim 0.02 \mathrm{~m}$ (i.e. $\sim 30 \%$ ). Whereas, in case of $50 \%$ emulsions, the increase of a surfactant fraction till 5 vol\% caused rising of the height $h_{i m}$ by $75 \%$, viz. from 0.04 to $0.07 \mathrm{~m}$ (Table II, Fig. 3-Fig. 4).

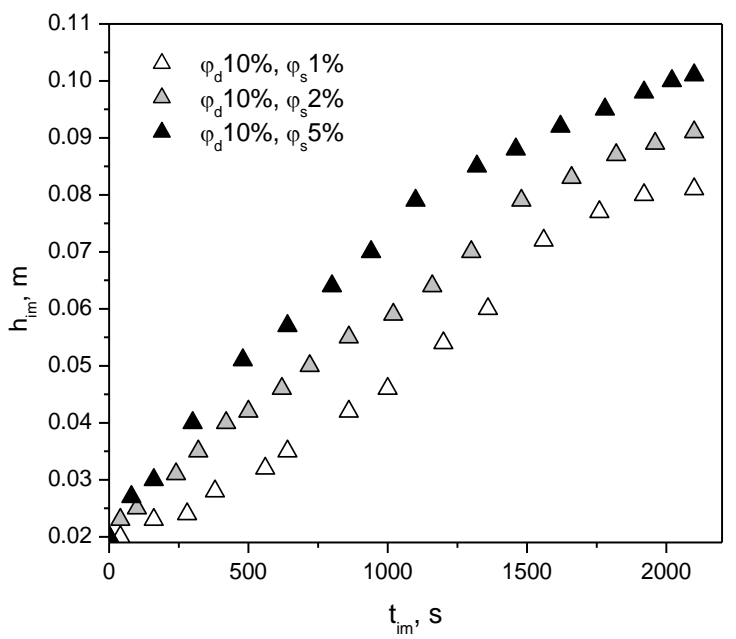

Fig. 3. Changes of an imbibed liquid height vs time for emulsions with different fractions of a surfactant $\left(\varphi_{d}=10 \mathrm{vol} \%\right)$.

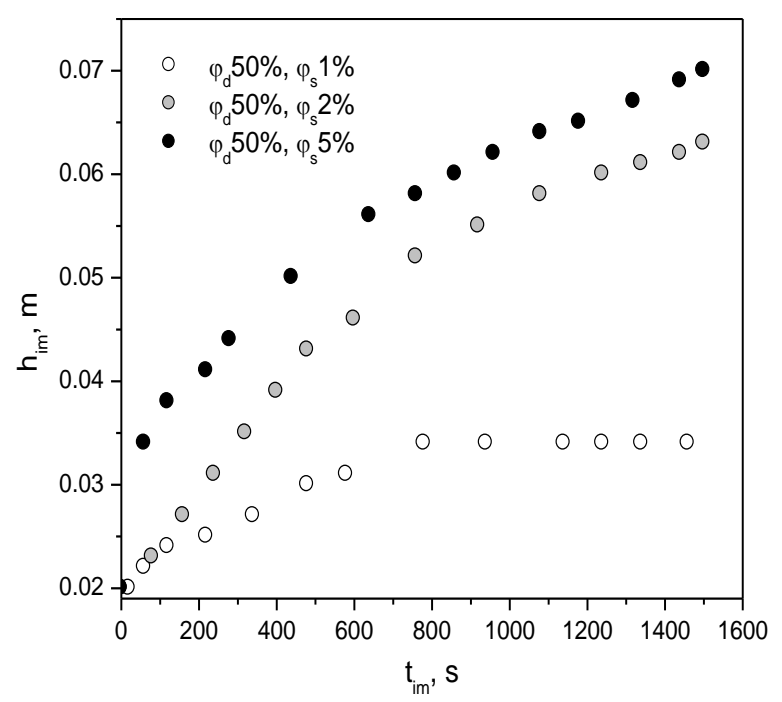

Fig. 4. Changes of an imbibed liquid height vs time for emulsions with different fractions of a surfactant $\left(\varphi_{d}=50 \mathrm{vol} \%\right)$.

To sum up, the concentration of the added emulsifier influenced on the kinetics of the imbibition process, especially in case of emulsions with $\varphi_{d} \geq 30 \mathrm{vol} \%$.

Additionally, the increase of an imbibed emulsion mass with the rising of height of its penetration was defined. The data obtained for emulsions with the emulsifier concentrations of 1 vol\% and 5 vol\% were shown in Fig. 5-Fig. 6.

For 50 vol\% emulsions, there was a difference in an amount of the imbibed permeants as well as the height of their penetration in the sorptive material (Table II-III, Fig. 4). However, as shown in Fig. 5-Fig. 6, the rate of the discussed process was the same for both represented emulsions stabilized by the different amount of the surfactant.

In case of the dispersed phase concentration of $30 \mathrm{vol} \%$, the rate of the imbibed mass change versus the height decreased for emulsions with $\varphi_{s}=5 \mathrm{vol} \%$ in comparison with another type (Fig. 5-Fig. 6). In contrast, the highest values of the maximal imbibed mass and height of penetration were 
obtained for this 30 vol\% emulsion (Table II-III, Fig. 5-Fig. 6 ). The slowing down was observed after $h_{i m} \geq 0.04 \mathrm{~m}$. The tendency revealed in case of 30 vol\% emulsions was also typical for $10 \%$ emulsions, but at $h_{i m} \geq 0.02 \mathrm{~m}$ (Fig. 5-Fig. 6).

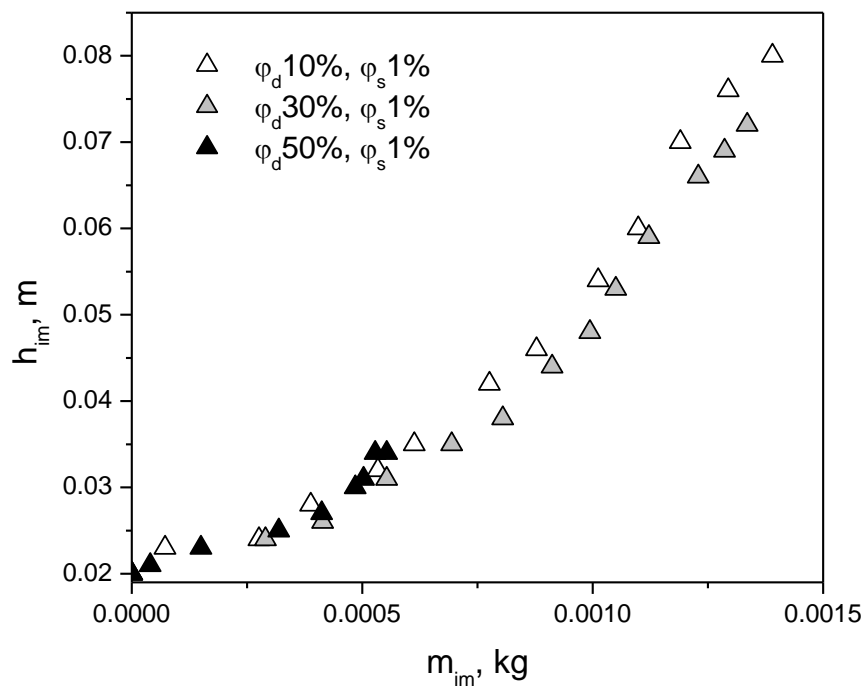

Fig. 5. Changes of an imbibed mass in a sorbent vs increase of front height for emulsions with different dispersed phase concentration $\left(\varphi_{s}=1 \mathrm{vol} \%\right)$.

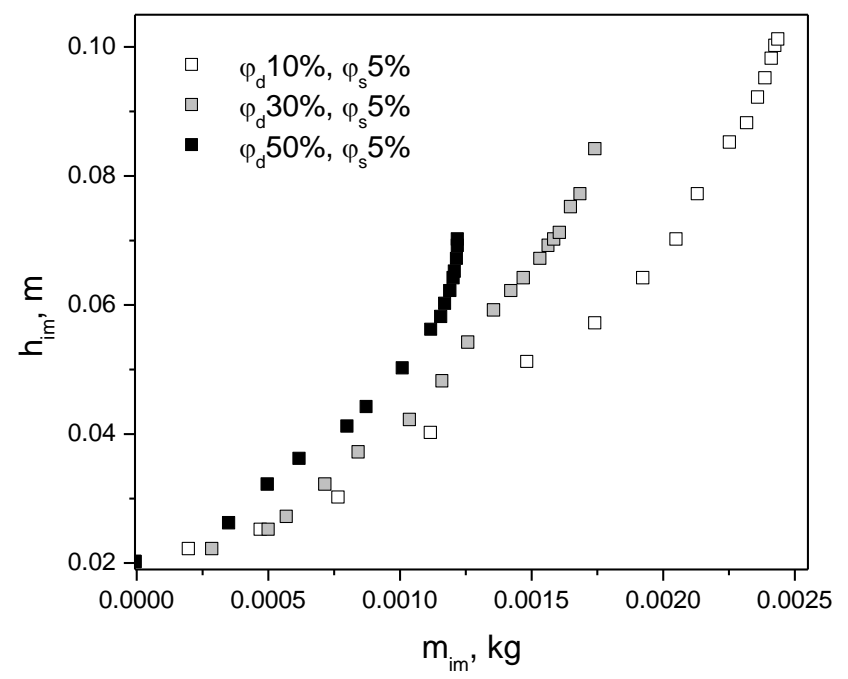

Fig. 6. Changes of an imbibed mass in a sorbent vs increase of front height for emulsions with different dispersed phase concentration $\left(\varphi_{s}=5 \mathrm{vol} \%\right)$.

To conclude, the rate of an imbibed mass increased with the rise of a liquid front height, depended significantly on an amount of the added non-ionic surfactant, but only in case when its concentration was more than 1 vol\% and the dispersed phase in an emulsion was lower than $50 \mathrm{vol} \%$.

\section{Changes of the Dispersed Phase Concentrations versus the Height}

The changes of the dispersed phase concentrations versus the height of an imbibed emulsion front are shown in Fig. 7-9. On the graphs, the vertical axis represents the normalized values of the dispersed phase concentration which were calculated as a ratio $\varphi_{i m} / \varphi_{d}$ where $\varphi_{i m}$ is defined as the experimentally obtained value of the inner phase concentration in an imbibed emulsion.

For all investigated liquids, the obtained concentrations of dispersed phase were lower in case of $\varphi_{s}=1$ vol\%, and consequently, the highest were observed for $\varphi_{s}=5 \mathrm{vol} \%$.

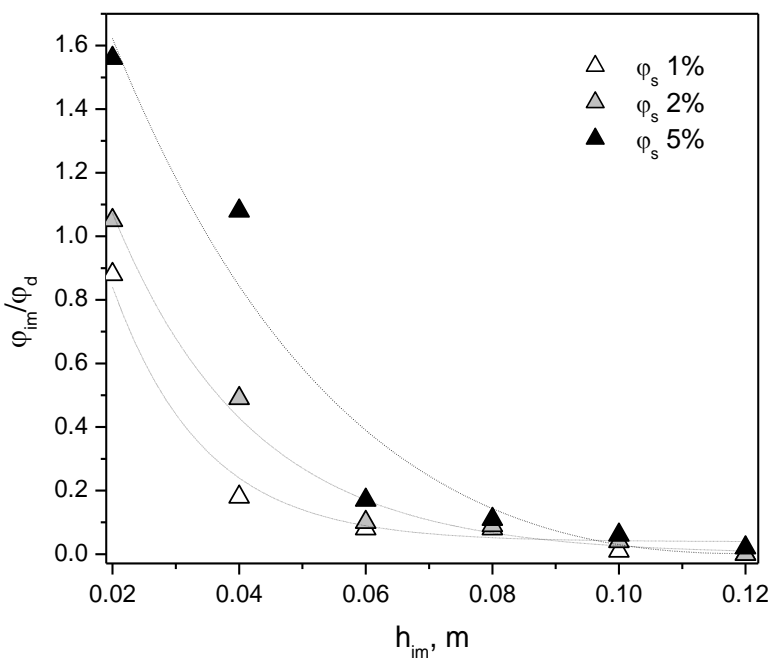

Fig. 7. Changes of the emulsions concentration vs height for different fractions of surfactant $\left(\varphi_{d}=10 \mathrm{vol} \%\right)$.

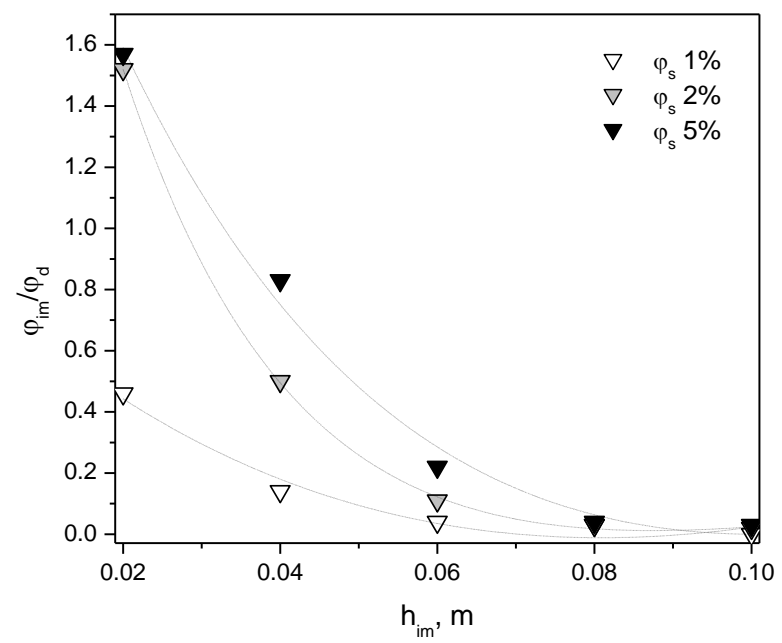

Fig. 8. Changes of the emulsions concentration vs height for different fractions of surfactant $\left(\varphi_{d}=30 \mathrm{vol} \%\right)$.

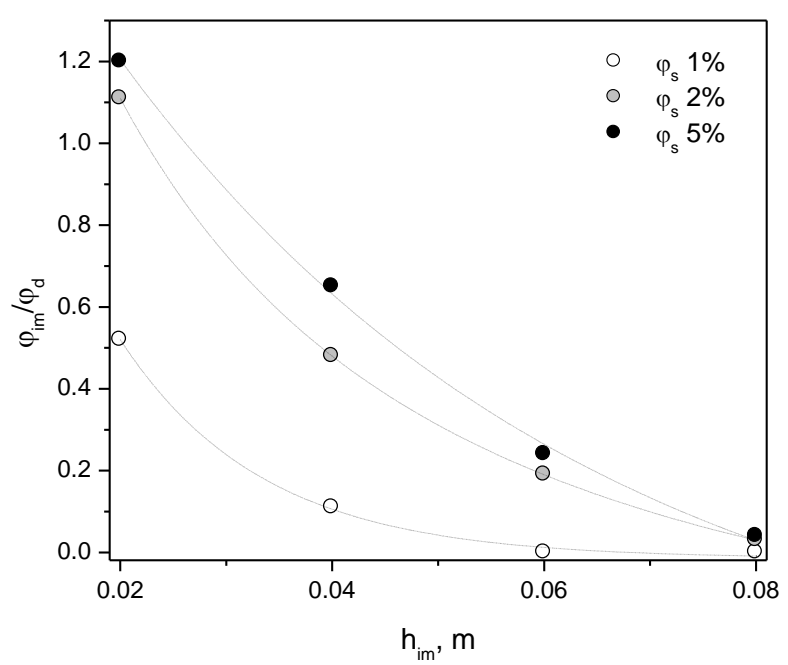

Fig. 9. Changes of the emulsions concentration vs height for different fractions of surfactant $\left(\varphi_{d}=50 \mathrm{vol} \%\right)$.

At the height $h_{i m} \leq 0.02 \mathrm{~m}$, the concentration of an imbibed emulsion $\varphi_{\text {im }}$ exceeded its initial value $\varphi_{d}$, but only if the surfactant was added in the amount of 2 vol\% or 5 vol\%. However, at $h_{i m}=0.08 \mathrm{~m}$, the obtained values of the dispersed phase concentration were almost identical for emulsions with different surfactant fractions, but the same fraction of the inner phase (Fig. 7-Fig. 9). 
In case of $30 \%$ and $50 \%$ emulsions, the curves describing the changes of a concentration, beside $\varphi_{s}=1 \mathrm{vol} \%$, were relatively close to each other (Fig. 7-Fig. 9). It proves that for emulsions with a higher dispersed phase concentration, i.e. $\varphi_{d} \geq 30 \mathrm{vol} \%$, the increase of the surfactant concentration had little effect on the investigated process.

To sum up, the augmentation of a non-ionic surfactant fraction up to $5 \mathrm{vol} \%$, provided the increasing of emulsions concentration versus the height of their penetration compare to others with $\varphi_{s}<5 \mathrm{vol} \%$.

\section{Changes of the Emulsion Viscosity versus the Height}

The observed changes of the dispersed phase concentration with the height can influence on viscosity of a penetrating emulsion and therefore, its penetration into porous structures. The obtained results concerning changes of the emulsions viscosity versus the height are plotted in Fig. 10-Fig. 12.

The effect of the emulsifier addition in the amount of $5 \mathrm{vol} \%$ was relative increasing of an imbibed emulsion viscosity compare to its initial value. This phenomenon was observed at height $h_{i m} \leq 0.02 \mathrm{~m}$ and can be explained by the increase of the imbibed emulsion concentration as shown in Fig. 7-Fig. 9.

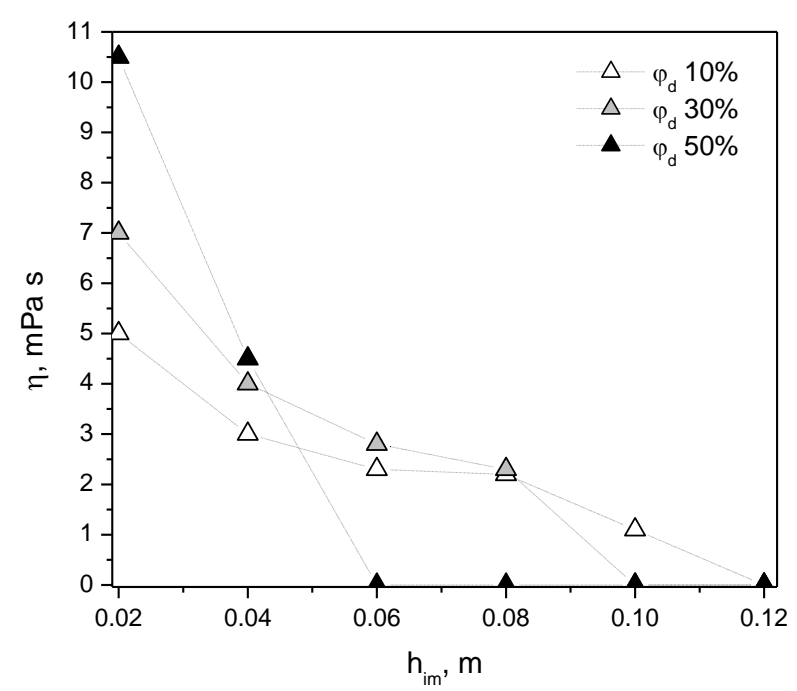

Fig. 10. Changes of the viscosity vs height for emulsions with different inner phase concentrations $\left(\varphi_{s}=1\right.$ vol $\left.\%\right)$.

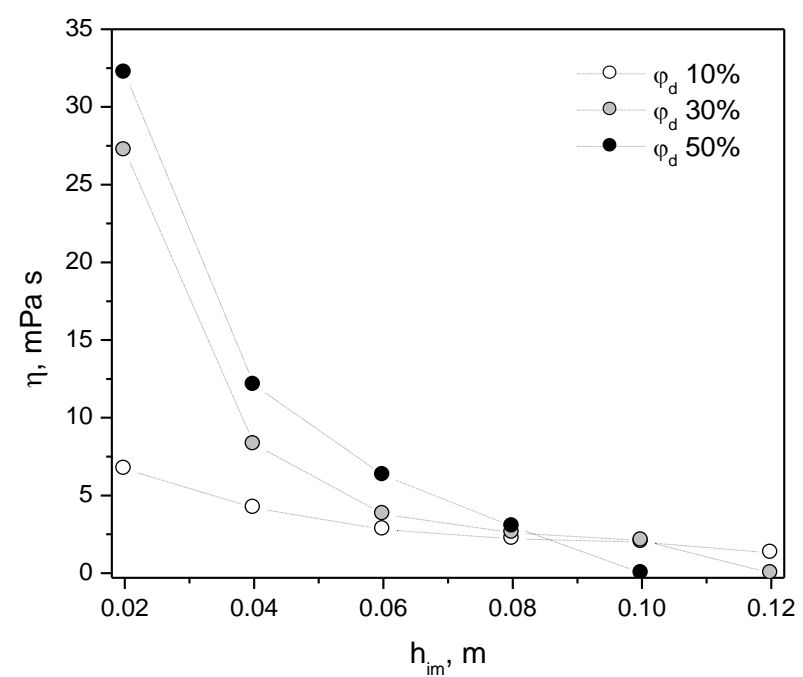

Fig. 11. Changes of the viscosity vs height for emulsions with different inner phase concentrations $\left(\varphi_{s}=2 \mathrm{vol} \%\right)$.

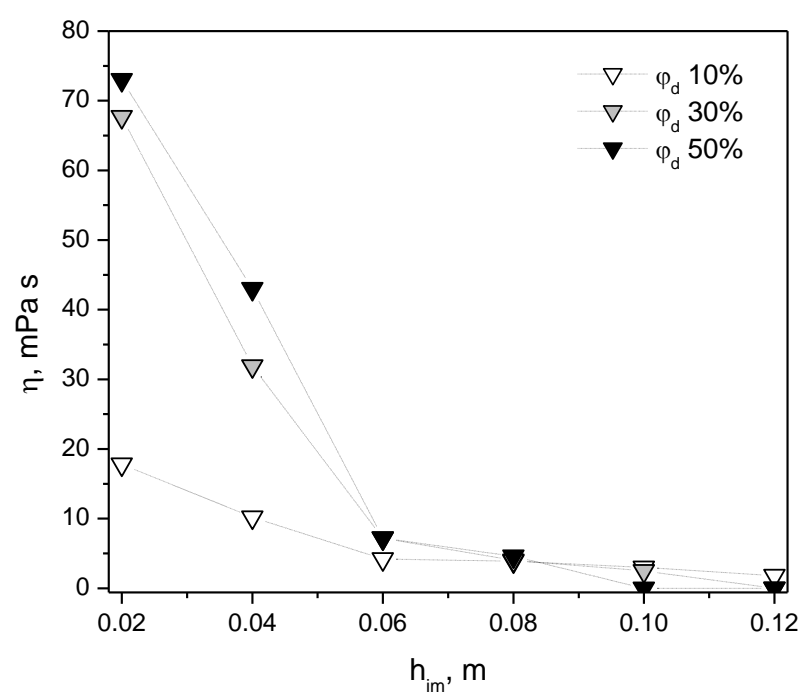

Fig. 12. Changes of the viscosity vs height for emulsions with different inner phase concentrations $\left(\varphi_{s}=5 \mathrm{vol} \%\right)$.

However, at $h_{i m} \geq 0.02 \mathrm{~m}$, the viscosity of an imbibed emulsion decreased with the rising of their front height in a sorbent. This tendency was typical for all investigated two-phase liquids.

There is a significant difference between the viscosity changes versus the height for emulsions with $\varphi_{s} \geq 2$ vol\% compare to the emulsions with lower fraction of the surfactant. At $h_{i m} \geq 0.08 \mathrm{~m}$, the obtained viscosity values were almost the same for all emulsions with surfactant concentration of 2 vol $\%$ and $5 \mathrm{vol} \%$. In case of $\varphi_{s} \geq 1 \mathrm{vol} \%$, higher results were observed for $50 \%$ emulsions, but only at the height less than $0.04 \mathrm{~m}$.

To sum up, the changes of viscosity as the opposite force to the capillary suction-pressure, can influence on the imbibition process, and consequently, should be taken into consideration within its description and modeling.

\section{CONCLUSION}

To sum up, an amount of the added non-ionic surfactant influenced on rheological properties of the investigated liquids. For example, the emulsions with a low dispersed phase concentration, i.e. $\varphi_{d}<30 \mathrm{vol} \%$, and the surfactant volume fraction of $\varphi_{s}<2$ vol\% behaved as Newtonian liquids, while at $\varphi_{d} \geq 30 \mathrm{vol} \%$ and $\varphi_{s} \geq 2$ vol\%, they exhibited properties of non-Newtonian liquids.

The process of porous structures imbibition with surfactant-stabilized emulsions depended on both the initial concentrations of the inner phase and an emulsifying agent. It revealed the increasing of mass and height of an imbibed emulsion with the raise of the surfactant fraction. In contrast, investigated parameters decreasing was observed simultaneously with an augmentation of the dispersed phase concentration, and the supposed reason for this can be the augmentation of viscous drag force.

Moreover, for emulsions with $\varphi_{d}<50 \mathrm{vol} \%$ and $\varphi_{s}>1 \mathrm{vol} \%$, the rate of the imbibed mass increase with the height of an emulsion penetration, depended significantly on a concentration of the used non-ionic emulsifying agent.

The obtained results also allowed to conclude that at the 
$h_{i m} \geq 0.02 \mathrm{~m}$, the inner phase concentration and viscosity of an imbibed emulsion decreased versus the height of its front in a porous medium in all investigated cases.

The observed variation of the dispersed phase concentration as well as the viscosity of the penetrating multiphase liquids can be a factor influencing on a process of their penetration thought porous oleophilic/hydrophobic structures.

\section{ACKNOWLEDGMENT}

The authors would like to acknowledge the support provided by the companies $\operatorname{Sintac}^{\circledR}-$ Polska Sp.z o.o.j.v., Warsaw (Poland) and PCC Exol SA., Brzeg Dolny (Poland).

\section{REFERENCES}

[1] R. Masoodi and K. M. Pillai, "Darcy's law-based model for wicking in paper-like swelling porous media," AIChE Journal, vol. 56, no. 9, pp. 2257-2267, Jan. 2010.

[2] M. Alava, M. Dube, and M. Rost, "Imbibition in disordered media," Advances in Physics, vol. 53, no. 2, pp. 83-175, Feb. 2004.

[3] B. V. Zhmud, F. Tiberg, and K. J. Hallstensson, "Dynamics of capillary rise," Journal of Colloid and Interface Science, vol. 228, no. 2, pp. 263-269, Aug. 2000.

[4] P. K. Chatterjee and B. S. Gupta, Absorbent Technology, 1st ed. Amsterdam: Elsevier B.V., 2002, ch. 2, pp. 78-79.

[5] H. T. Xue, Z. N. Fang, Y. Yang, J. P. Huang, and L. W. Zhou, "Contact angle determined by spontaneous dynamic capillary rises with hydrostatic effects: Experiment and theory," Chemical Physics Letters, vol. 432 , no. $1-3$, pp. 326-330, Oct. 2006.

[6] A. Siebold, M. Nardin, J. Schultz, A. Walliser, and M. Oppliger, "Effect of dynamic contact angles on capillary rise phenomena," Colloids and Surfaces A: Physicochemical and Engineering Aspects, vol. 161, no. 1, pp. 81-87, Jan. 2000.

[7] R. M. Digilov, "Capillary rise of a non-Newtonian power law liquid: Impact of the fluid rheology and dynamic contact angle," Langmuir, vol. 24, no. 23, pp. 13663-13667, Nov. 2008.

[8] A. Hamraoui and T. Nylander, "Analytical approach for the Lucas-Washburn equation," Journal of Colloid and Interface Science, vol. 250, no. 2, pp. 415-421, Feb. 2002.

[9] R. Masoodi, K. M. Pillai, and P. P. Varanasi, "Darcy's law based models for liquid absorption in polymer wicks," AIChE Journal, vol. 53, no. 11, pp. 2769-2782, Sept. 2007.

[10] T. Dang-Vu and J. Hupka, "Characterization of porous materials by capillary rise method," Physicochemical Problems of Mineral Processing, vol. 39, pp. 47-65, May 2005.

[11] J. Cai, X. Hu, D. C. Standnes, and L. You, "An analytical model for spontaneous imbibition in fractal porous media including gravity," Colloids and Surfaces A: Physicochemical and Engineering Aspects, vol. 414, pp. 228-233, Nov. 2012.

[12] B. P. Blink and S. O. Lumson, "Influence of particle wettability on the type and stability of surfactant-free emulsions," Langmuir, vol. 16, pp. 8622-8631, June 2000

[13] J. Bibette and F. Leal-Calderon, "Surfactant-stabilized emulsions," Current Opinion in Colloid \& Interface Science, vol. 1, no. 6, pp. 746-751, Dec. 1996.

[14] B. P. Blinks, "Particles as surfactants—similarities and differences," Current Opinion in Colloid \& Interface Science, vol. 7, no. 1-2, pp. 21-41, Mar. 2002.

[15] F. Leal-Calderon and V. Schmitt, "Solid-stabilized emulsions," Current Opinion in Colloid \& Interface Science, vol. 13, no. 4, pp. 217-227, Aug. 2008.

[16] J. W. Kim, D. Lee, H. C. Shum, and D. A. Weitz, "Colloid surfactant for emulsion stabilization," Advanced Materials, vol. 20, no. 17, pp. 3239-3243, July 2008.

[17] K. Rahn-Chiquea, A. M. Puertas, M. S. Romero-Canob, C. Rojasa, and G. Urbina-Villalbaa, "Nanoemulsion stability: Experimental evaluation of the flocculation rate from turbidity measurements," Advances in Colloid and Interface Science, vol. 178, pp. 1-20, Oct. 2012.
[18] T. Tadros, P. Izguierdo, J. Esquena, and C. Solans, "Formation and stability of nano-emulsions," Advances in Colloid and Interface Science, vol. 108-109, pp. 303-318, May 2004.

[19] J. M. Gutierrez, C. Gonzalez, A. Maestro, I. Sole, C. M. Pey, and J. Nolla, "Nano-emulsions: New applications and optimization of their preparation," Current Opinion in Colloid \& Interface Science, vol. 13 no. 4, pp. 245-251, Aug. 2008.

[20] B. P. Binks and J. A. Rodrigues, "Synergistic Interaction in Emulsions Stabilized by a Mixture of Silica Nanoparticles and Cationic Surfactant," Langmuir, vol. 23, no. 7, pp. 3626-3636, Feb. 2007.

[21] P. Izquierdo, J. Feng, J. Esquena, T. F. Tadros, J. C. Dederen, M. J. Garcia, N. Azemar, and C. Solans, "The influence of surfactant mixing ratio on nano-emulsion formation by the pit method," Journal of Colloid and Interface Science, vol. 283, no. 1, pp. 388-394, May 2005.

[22] O. Behrend, K. Ax, and H. Schubert, "Influence of continuous phase viscosity on emulsification by ultrasound," Ultrasonics Sonochemistry, vol. 7, no. 2, pp. 77-85, April 2000.

[23] J. Sęk, O. Shtyka, and K. Szymczak, "Modeling of the spontaneous polypropylene sorbents imbibition with emulsions," Journal of Environmental Engineering and Landscape Management, vol. 23, no. 3, pp. 83-93, Dec. 2014.

[24] C. Lemarchand, P. Couvreur, C. Vauthier, D. Costantini, and R. Gref "Study of emulsion stabilization by graft copolymers using optical analyzer turbiscan," International Journal of Pharmaceutics, vol. 254, pp. 77-82, Mar. 2003

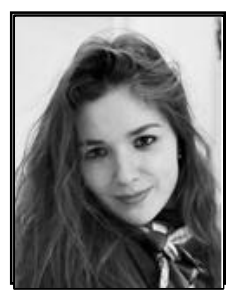

Olga S. Shtyka was born in Russia on May 15, 1987 She is a PhD student in chemical engineering, the Lodz University of Technology, Lodz, Poland, since 2012. She received her MSc. in ecology and ecological safety from the National Aviation University, Kiev. Ukraine, 2010

She worked as Assistant of Professor in the National Aviation University from 2010-2011, Consultant in a sphere of the environmental protection from 2010-2012, Auditor in a company "EcoGroup", Kyiv, and Internship in the Ministry of Emergency Situations of Ukraine in 2007. She took participation in more than 15 International conferences in Belgium, France, Sweden, Italy, Switzerland, Russia, Poland, Belarus, Lithuania and Ukraine and is coauthor of 8 articles. The field of the current research work: flow of multiphase liquids in porous structures, rheological properties of emulsions.

Ms. Shtyka is a member of the Commission on International Collaboration of the National Association of PhD students of Poland. She is a holder of the Rector scholarship for $\mathrm{PhD}$ students who are non-residents of European Union 2013/2016 and the High academic Achievement Scholarship 2015/2016. She was also holder of the scholarship of the President of Ukraine for the young scientists in 2009/2010.

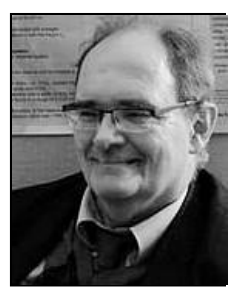

Jerzy P. Sęk was born in Lodz, Poland on July 11, 1950. He is Professor in chemical engineering, the Faculty of Process and Environment Engineering, the Lodz University of Technology, Lodz, Poland, since 2005. He received his DSc. Eng. in chemical engineering, from Institute of Chemical Engineering, the Lodz University of Technology, Lodz, Poland, in 1980.

He has been working as Professor of the Faculty of Process and Environment Engineering, the Lodz University of Technology since 2008; Associate Professor in the Institute of Chemical Engineering, the Lodz University of Technology from 1981 to 2008; Scientific researcher in the Department of Physical Technology, the University of Groningen, Groningen, Netherlands from 1988 to 1990; RWTH Aachen University, Aachen, Germany in 1986; Scientific researcher in the Delft University of Technology, Delft, Netherlands from 1982-1983. He is author and co-author of 69 publications and 7 monographies. His major field of the research work: flow of multi-phase liquids in porous structures; elusion of high viscous liquids; rheological properties of emulsions, their stability and numerical simulation of phases separation process

Prof. Sek is a secretary and member of the Board of the Polish Society of Technical Rheology since 1997. He received the Medal of the National Education Commission; Award of the Minister of Environmental Protection; Awards of the Minister of Education. Prof. Sęk is a recipient of the Bronze Cross of Merit (Poland) 